\title{
First-order symmetrizable hyperbolic formulations of Einstein's equations including lapse and shift as dynamical fields
}

\author{
Kashif Alvi \\ Theoretical Astrophysics, California Institute of Technology, Pasadena, CA 91125, USA \\ E-mail: kashif@tapir.caltech.edu
}

Received 26 April 2002, in final form 9 August 2002

Published 1 October 2002

Online at stacks.iop.org/CQG/19/5153

\begin{abstract}
First-order hyperbolic systems are promising as a basis for numerical integration of Einstein's equations. In previous work, the lapse and shift have typically not been considered part of the hyperbolic system and have been prescribed independently. This can be expensive computationally, especially if the prescription involves solving elliptic equations. Therefore, including the lapse and shift in the hyperbolic system could be advantageous for numerical work. In this paper, two first-order symmetrizable hyperbolic systems are presented that include the lapse and shift as dynamical fields and have only physical characteristic speeds.
\end{abstract}

PACS numbers: $0420,0425 \mathrm{D}$

\section{Introduction}

There has been considerable interest recently in first-order hyperbolic systems for Einstein's equations ([1-3] and references therein). These systems have been used in the past to prove that general relativity has a well-posed initial value formulation $[4,5]$. Much of the recent interest is based on the advantages that hyperbolic formulations offer to numerical simulations [6,7]. The main advantage is that imposing physical boundary conditions is much easier in the framework of a hyperbolic system than a non-hyperbolic one. This is especially true for boundary conditions inside a black-hole horizon [6,7]. Indeed, if the hyperbolic system has only physical characteristic speeds - that is, if the characteristic fields propagate only on the light cones of spacetime or normal to the time slices-then the boundary condition inside the horizon on fields propagating into the numerical grid has no effect on the dynamics outside the horizon ${ }^{1}$. Therefore, in this case, any convenient boundary condition can be imposed inside the horizon. This is a significant advantage when simulating black holes.

1 It is sufficient for the characteristic fields to propagate on or within the light cones for this to be true. 
It is particularly important to come up with stable numerical schemes to evolve black holes since simulations of black-hole collisions have an important role to play in the detection and analysis of gravitational waves. These simulations will be used in several stages of data analysis for gravitational wave detectors such as the Laser Interferometer Gravitational-Wave Observatory. First, the simulations are expected to yield a bank of gravitational waveforms that will be used to detect the presence of a gravitational signal in the detector output. Once a signal has been detected, numerical simulations will be used to extract binary parameters, such as masses from the signal, to test general relativity, and to do other interesting physics.

Previous numerical work has generally been restricted to systems that do not treat the lapse and shift as dynamical fields, but rather take them to be external to the system and prescribe them independently. Freedom in choosing these gauge fields corresponds to freedom in choosing coordinates for spacetime. This freedom can be used for a variety of purposes, e.g., to prevent the occurrence of coordinate singularities and reduce coordinate shear [8], and to adapt the coordinate system to the particular problem under consideration. When simulating black holes, it is helpful to choose the shift so that numerical grid points do not fall into the holes. When simulating binary black holes, it may be advantageous to implement gauge conditions which generate corotating coordinates $[9,10]$.

Some of the favoured gauge choices in numerical relativity $[8,10]$ require solution of elliptic equations for the lapse and shift, which is expensive computationally. It would be more efficient to evolve the gauge fields as part of the hyperbolic system. However, it is important to keep some freedom in choosing the gauge in order to allow the coordinates to be adapted to fit specific needs. The purpose of this paper is to present two first-order symmetrizable hyperbolic systems which include the lapse and shift as dynamical fields and allow four functions of spacetime to be specified freely in the gauge prescription.

Previous work in this direction includes [11], in which the authors present a weakly hyperbolic system ${ }^{2}$ that incorporates the gauge fields in the system, and [12], in which the authors present a new class of dynamical gauge conditions which are not, however, part of a first-order hyberbolic system.

The first hyperbolic system presented in this paper is based on the work of Fischer and Marsden [4]; it uses generalized harmonic coordinates and evolves 50 fields. It is promising as a basis for numerical work. The second system is based on the work of Kidder et al [3] and Lindblom and Scheel [13]; it evolves 70 fields. This system is not practical for numerical implementation. Its main use is theoretical: it allows one to show that any solution to Einstein's equations in any gauge can be obtained using hyperbolic evolution of the entire metric, including the gauge fields. Both systems have only physical characteristic speeds.

In this paper, Greek indices range over 0-3 and Latin indices over 1-3. The sign conventions are those of [14] with $G=c=1$. The analysis of this paper is done within the framework of a $3+1$ split of spacetime (see, e.g., $[14,15]$ ). In this framework, the spacetime metric is expressed as

$$
g_{\mu \nu}=\left(\begin{array}{cc}
-\alpha^{2}+\beta_{k} \beta^{k} & \beta_{j} \\
\beta_{i} & \gamma_{i j}
\end{array}\right),
$$

and the inverse 4-metric as

$$
g^{\mu \nu}=\frac{1}{\alpha^{2}}\left(\begin{array}{cc}
-1 & \beta^{j} \\
\beta^{i} & \alpha^{2} \gamma^{i j}-\beta^{i} \beta^{j}
\end{array}\right),
$$

\footnotetext{
2 I refer to the full system including lapse and shift as dynamical fields; if the shift is considered a fixed spacetime function and not a dynamical field, then the system becomes strongly hyperbolic.
} 
where $\alpha$ is the lapse, $\beta^{i}$ is the shift, $\gamma_{i j}$ is the spatial 3 -metric with inverse $\gamma^{i j}$, and $\beta_{i}=\gamma_{i j} \beta^{j}$. The unit normal to the time slices is denoted by $n^{\mu}$.

I restrict attention in this paper to the vacuum Einstein equations.

\section{System I}

\subsection{Fischer-Marsden system}

Let us first briefly review the Fischer-Marsden system [4] for Einstein's equations. They employ the 50 fields $g_{\mu \nu}, \tilde{k}_{\mu \nu}=\partial_{t} g_{\mu \nu}$, and $d_{i \mu \nu}=\partial_{i} g_{\mu \nu}$. Using harmonic coordinates, they reduce the vacuum Einstein equations $R_{\mu \nu}=0$ to the following first-order symmetric hyperbolic system:

$$
\begin{aligned}
& \partial_{t} g_{\mu \nu}=\tilde{k}_{\mu \nu}, \\
& -g^{00} \partial_{t} \tilde{k}_{\mu \nu}-2 g^{0 i} \partial_{i} \tilde{k}_{\mu \nu}-g^{i j} \partial_{i} d_{j \mu \nu}=-2 \tilde{H}_{\mu \nu}, \\
& g^{i j} \partial_{t} d_{j \mu \nu}-g^{i j} \partial_{j} \tilde{k}_{\mu \nu}=0,
\end{aligned}
$$

where $\tilde{H}_{\mu \nu}$ is a function of the fields $g_{\mu \nu}, \tilde{k}_{\mu \nu}, d_{i \mu \nu}$ only and not their derivatives. This system is obtained by setting to zero a reduced form of the Ricci tensor that is equal to the full Ricci tensor in harmonic coordinates. Using earlier work of Choquet-Bruhat ([16] and references therein), Fischer and Marsden show that if the initial data for (2.1) satisfy the harmonic coordinate condition and the constraint equations, then the solution of (2.1) corresponding to these initial data continues to satisfy the harmonic coordinate condition off the initial hypersurface. Therefore, a solution of (2.1) is also a solution of the vacuum Einstein equations.

The Fischer-Marsden system (2.1) has two drawbacks when considered as a basis for numerical integration of Einstein's equations. The first is the restriction to harmonic coordinates: this eliminates the freedom to choose coordinates best suited for the physical problem at hand. While harmonic coordinates have been used successfully in some previous work ([17] and references therein) and are being strongly advocated for a wide variety of applications [17], it has not yet been established whether they are useful for simulating blackhole collisions, for example.

The second drawback is that the Fischer-Marsden system has nonphysical characteristic speeds. As discussed above, systems with only physical characteristic speeds are better suited for numerical relativity, especially for black-hole simulations [6, 7]. The characteristic speeds of the Fischer-Marsden system can be calculated as follows: first write (2.1) in the form

$$
\partial_{t} u+A^{i}\left(t, x^{j}, u\right) \partial_{i} u=F\left(t, x^{j}, u\right)
$$

where $u$ is a column vector composed of the fields $\left(u=\left(g_{\mu \nu}, \tilde{k}_{\mu \nu}, d_{i \mu \nu}\right)^{T}\right.$ for the FischerMarsden system), and the matrices $A^{i}$ and column vector $F$ can depend on space and time and on the fields but not their derivatives. Pick a unit spatial covector $\xi_{i}$ (i.e., $\gamma^{i j} \xi_{i} \xi_{j}=1$ ) and compute the eigenvalues $\lambda$ of the matrix $A^{i} \xi_{i} ; \lambda$ are the characteristic speeds in the direction $\xi_{i}$. For physical characteristic speeds, we require $\lambda=-\beta^{i} \xi_{i},-\beta^{i} \xi_{i} \pm \alpha$ (see, e.g., [3]). However, the Fischer-Marsden system has $\lambda=0,-\beta^{i} \xi_{i} \pm \alpha$.

\subsection{Generalized harmonic coordinates}

In this paper, I modify the Fischer-Marsden system to eliminate nonphysical characteristic speeds and generalize it to include a broader range of coordinate systems. Let us begin by defining $\Gamma^{\mu}=g^{\alpha \beta} \Gamma_{\alpha \beta}^{\mu}$ and $\Gamma_{\mu}=g_{\mu \nu} \Gamma^{\nu}$, where $\Gamma_{\alpha \beta}^{\sigma}$ are the Christoffel symbols associated with the metric $g_{\mu \nu}$ and the coordinates $x^{\mu}$. The Ricci tensor can be written as [18]

$$
R_{\mu \nu}=\tilde{R}_{\mu \nu}+\nabla_{(\mu} \Gamma_{\nu)},
$$


where

$$
\tilde{R}_{\mu \nu}=-\frac{1}{2} g^{\alpha \beta} \partial_{\alpha} \partial_{\beta} g_{\mu \nu}+H_{\mu \nu}(g, \partial g),
$$

and

$$
H_{\mu \nu}=g^{\alpha \beta} g^{\rho \sigma}\left(\partial_{\alpha} g_{\mu \rho}\right)\left(\partial_{\beta} g_{\nu \sigma}\right)-g^{\rho \alpha} g^{\sigma \beta} \Gamma_{\mu \rho \sigma} \Gamma_{\nu \alpha \beta} .
$$

I generalize harmonic coordinates using Friedrich's gauge source functions $[5,6]$ by setting

$$
\Gamma^{\mu} \equiv-\nabla_{\alpha} \nabla^{\alpha} x^{\mu}=f^{\mu}\left(t, x^{j}\right),
$$

where the coordinates $x^{\mu}$ are treated as scalar fields in the expression $\nabla_{\alpha} \nabla^{\alpha} x^{\mu}$, and $f^{\mu}$ are arbitrary but predetermined functions of space and time. These functions can be used to tailor the coordinates to fit specific needs.

Consider the reduced equations obtained by setting

$$
\tilde{R}_{\mu \nu}+\nabla_{(\mu} f_{\nu)}=0
$$

where $f_{\mu}=g_{\mu \nu} f^{\nu}$. Equation (2.7) will be used to write a first-order symmetrizable hyperbolic system in section 2.3. Hence we must show that a solution to (2.7) yields a solution to the vacuum Einstein equations $R_{\mu \nu}=0$ under appropriate conditions. I follow an argument given by Friedrich [5, 6] which is based on earlier work by Choquet-Bruhat ([16] and references therein).

Let $g_{\mu \nu}$ be a solution to (2.7). Compute $\Gamma^{\mu}$ and $R_{\mu \nu}$ from $g_{\mu \nu}$, and let $h^{\mu}=\Gamma^{\mu}-f^{\mu}$. Then $R_{\mu \nu}=\tilde{R}_{\mu \nu}+\nabla_{(\mu} \Gamma_{\nu)}=\nabla_{(\mu} h_{\nu)}$ where $h_{\mu}=\Gamma_{\mu}-f_{\mu}$. The Einstein tensor is

$$
G_{\mu \nu}=R_{\mu \nu}-\frac{R}{2} g_{\mu \nu}=\nabla_{(\mu} h_{\nu)}-\frac{1}{2} g_{\mu \nu} \nabla_{\alpha} h^{\alpha},
$$

and the contracted Bianchi identities $\nabla^{\mu} G_{\mu}{ }^{\nu}=0$ imply

$$
\nabla^{\mu} \nabla_{\mu} h^{v}+R_{\mu}^{v} h^{\mu}=0
$$

which is the subsidiary equation derived by Friedrich [5, 6]. Since this is a linear homogeneous wave equation for $h^{\mu}$, we conclude that if $h^{\mu}=0$ and $\nabla_{\nu} h^{\mu}=0$ on the initial hypersurface, then $h^{\mu}=0$ in a neighbourhood of the initial hypersurface. This implies $R_{\mu v}=\nabla_{(\mu} h_{v)}=0$ in this neighbourhood. So $g_{\mu \nu}$ is a solution to the vacuum Einstein equations in a neighbourhood of the initial hypersurface. This solution is obtained in coordinates satisfying $\Gamma^{\mu}=f^{\mu}$.

We therefore need to ensure

$$
\begin{aligned}
& {\left[\Gamma^{\mu}-f^{\mu}\right]_{t=0}=0,} \\
& {\left[\nabla_{v}\left(\Gamma^{\mu}-f^{\mu}\right)\right]_{t=0}=0,}
\end{aligned}
$$

where the time slice $t=0$ represents the initial hypersurface. Given a spatial 3-metric $\gamma_{i j}$ and an extrinsic curvature $K_{i j}$ that satisfy the constraint equations, we will construct initial data for our system such that (2.10) is satisfied. Equation (2.11) will then follow from the constraint equations. This will be discussed in detail in section 2.4.

\subsection{System I}

Define the fields

$$
\begin{aligned}
& k_{\mu \nu}=\partial_{t} g_{\mu \nu}-\beta^{j} \partial_{j} g_{\mu \nu}, \\
& d_{i \mu \nu}=\partial_{i} g_{\mu \nu} .
\end{aligned}
$$


Here and throughout this section, $\beta^{i}$ will be considered convenient shorthand for $-g^{0 i} / g^{00}$, and similarly $\alpha$ for $\left(-g^{00}\right)^{-1 / 2}$. The new field $k_{\mu \nu}$ is a replacement for $\tilde{k}_{\mu \nu}$ and has been introduced to eliminate nonphysical characteristic speeds.

The first-order symmetrizable hyperbolic system presented in this section is based on the 50 fields $g_{\mu \nu}, k_{\mu \nu}$ and $d_{i \mu \nu}$. The definition (2.12) yields an expression for $\partial_{t} g_{\mu \nu}$ in terms of the 50 fields and their first spatial derivatives. An expression for $\partial_{t} d_{i \mu \nu}$ is obtained through equality of mixed partials: $\partial_{t} d_{i \mu \nu}=\partial_{i} \partial_{t} g_{\mu \nu}=\partial_{i}\left(k_{\mu \nu}+\beta^{j} d_{j \mu \nu}\right)$. Finally, an expression for $\partial_{t} k_{\mu \nu}$ is obtained from the reduced equation (2.7). To summarize, we have the first-order system

$\partial_{t} g_{\mu \nu}+\frac{g^{0 i}}{g^{00}} \partial_{i} g_{\mu \nu}=k_{\mu \nu}$

$\partial_{t} k_{\mu \nu}+\frac{g^{0 i}}{g^{00}} \partial_{i} k_{\mu \nu}+\frac{\gamma^{i j}}{g^{00}} \partial_{i} d_{j \mu \nu}=-\frac{\gamma^{i j}}{g^{00}} g^{0 \alpha} d_{i \mu \nu} k_{\alpha j}+\frac{2}{g^{00}}\left[H_{\mu \nu}+\partial_{(\mu} f_{\nu)}-\Gamma_{\mu \nu}^{\alpha} f_{\alpha}\right]$,

$\partial_{t} d_{i \mu \nu}+\frac{g^{0 j}}{g^{00}} \partial_{j} d_{i \mu \nu}-\partial_{i} k_{\mu \nu}=\frac{\gamma^{j k}}{g^{00}} g^{0 \alpha} d_{j \mu \nu} d_{i \alpha k}$

where $\gamma^{i j}=\left(g^{00}\right)^{-2}\left(g^{00} g^{i j}-g^{0 i} g^{0 j}\right)$ is the inverse of the 3 -metric $\gamma_{i j}$. In (2.15), $H_{\mu \nu}$ is to be expressed via (2.5) in terms of the fields only and not their derivatives (using (2.12) and (2.13)). In addition, in (2.14)-(2.16), the inverse 4-metric is considered to be a function of $g_{\mu \nu}$ and not a fundamental field. In deriving these expressions, I have used the relation

$$
\partial_{\alpha} g^{\mu \nu}=-g^{\mu \theta} g^{\nu \lambda} \partial_{\alpha} g_{\theta \lambda} .
$$

The system (2.14)-(2.16) will be called system I.

\subsection{Initial data}

It remains to specify how to set initial data for system I to ensure (2.10) and (2.11) are satisfied. Begin with a solution $\left(\gamma_{i j}, K_{i j}\right)$ of the constraint equations, where $K_{i j}$ represents the extrinsic curvature of the initial hypersurface. First set $g_{i j}=\gamma_{i j}$. We are free to choose $g_{0 \mu}$ on the initial hypersurface as long as $g_{00}<g_{0 i} g_{0 j} \gamma^{i j}$. This requirement is equivalent to $\alpha^{2}>0$ and implies $g^{00}<0$. Freedom in choosing $g_{0 \mu}$ corresponds to freedom in choosing the lapse and shift at $t=0$.

We now have $\left.g_{\mu \nu}\right|_{t=0}$. Next set $d_{i \mu \nu}=\left.\partial_{i} g_{\mu \nu}\right|_{t=0}$. The final step is to fill in $k_{\mu \nu}$ from $K_{i j}$ and the requirement (2.10). The extrinsic curvature can be expressed as

$$
K_{i j}=-\frac{1}{2 \alpha}\left(\partial_{t} \gamma_{i j}-\beta^{k} \partial_{k} \gamma_{i j}-2 \gamma_{k(i} \partial_{j)} \beta^{k}\right) .
$$

From this we deduce

$$
k_{i j}=-2 \alpha K_{i j}+2 g_{k(i} \partial_{j)} \beta^{k},
$$

which can be used to fill in $k_{i j}$ at $t=0$.

The quantities $k_{0 \mu}$ are obtained from the requirement (2.10). Writing out $\Gamma^{\mu}$ in terms of the metric and its first derivatives, we obtain

$$
\begin{aligned}
& \Gamma^{0}=-\alpha^{-3}\left(\partial_{t} \alpha-\beta^{i} \partial_{i} \alpha+\alpha^{2} K\right), \\
& \Gamma^{i}=-\alpha^{-2}\left(\partial_{t} \beta^{i}-\beta^{j} \partial_{j} \beta^{i}\right)+\alpha^{-3}\left(\partial_{t} \alpha-\beta^{j} \partial_{j} \alpha+\alpha^{2} K\right) \beta^{i}-\alpha^{-1} \gamma^{i j} \partial_{j} \alpha+{ }^{(3)} \Gamma^{i}{ }_{j k} \gamma^{j k},
\end{aligned}
$$


where $K=\gamma^{i j} K_{i j}$, and ${ }^{(3)} \Gamma^{i}{ }_{j k}$ are the Christoffel symbols associated with the 3 -metric $\gamma_{i j}$ and the spatial coordinates $x^{j}$. Setting $\Gamma^{\mu}=f^{\mu}$ gives us expressions for $\partial_{t} \alpha$ and $\partial_{t} \beta^{i}$ which we use to fill in $k_{0 \mu}$ at $t=0$ :

$$
\begin{aligned}
& k_{0 i}=B_{i}+\beta^{j} k_{i j}, \\
& k_{00}=2 \alpha^{3}\left(\alpha f^{0}+K\right)+2 \beta^{i} B_{i}+\beta^{i} \beta^{j} k_{i j},
\end{aligned}
$$

where

$$
B_{i}=-\alpha^{2}\left(g_{i \mu} f^{\mu}+\alpha^{-1} \partial_{i} \alpha-{ }^{(3)} \Gamma_{i j k} \gamma^{j k}\right) .
$$

The initial data for system I are now complete and satisfy the constraint equations

$$
\left.G_{\mu \nu} n^{\nu}\right|_{t=0}=0
$$

and the requirement (2.10). This in fact implies that the requirement (2.11) is satisfied. The argument follows earlier work [16] on the reduction of Einstein's equations using harmonic coordinates. From (2.8) and (2.25), we deduce

$$
2 n^{\nu} \nabla_{(\mu} h_{\nu)}-n_{\mu} \nabla_{\alpha} h^{\alpha}=0 .
$$

Here and in the remainder of the paragraph, all quantities are evaluated at $t=0$. We know $h^{\mu} \equiv \Gamma^{\mu}-f^{\mu}=0$ on the initial hypersurface, so $v^{\nu} \nabla_{\nu} h^{\mu}=0$ for any spatial vector $v^{\mu}$ (i.e., for $v^{\mu}$ satisfying $v^{\mu} n_{\mu}=0$ ). It remains to show $n^{\nu} \nabla_{\nu} h^{\mu}=0$. By contracting (2.26) with $v^{\mu}$, we obtain $v^{\mu} n^{\nu} \nabla_{\nu} h_{\mu}=0$. Furthermore, $\nabla_{\alpha} h^{\alpha}=-n^{\mu} n^{\nu} \nabla_{\mu} h_{\nu}$. Contracting (2.26) with $n^{\mu}$, we obtain $n^{\mu} n^{\nu} \nabla_{\mu} h_{\nu}=0$. It follows that $n^{\nu} \nabla_{\nu} h^{\mu}=0$ and so (2.11) is satisfied.

Therefore, a solution $\left(g_{\mu \nu}, k_{\mu \nu}, d_{i \mu \nu}\right)$ to system I with initial data as constructed above yields a solution $g_{\mu \nu}$ to the vacuum Einstein equations.

\subsection{Hyperbolicity of system I}

System I is symmetrizable hyperbolic. To see this, let $u=\left(g_{\mu \nu}, k_{\mu \nu}, d_{1 \mu \nu}, d_{2 \mu \nu}, d_{3 \mu \nu}\right)^{T}$ and write equations (2.14)-(2.16) in the form (2.2). This determines the $50 \times 50$ matrices $A^{i}$ to be

$$
A^{i}=\left(\begin{array}{ccccc}
-\beta^{i} I & 0 & 0 & 0 & 0 \\
0 & -\beta^{i} I & -\alpha^{2} \gamma^{i 1} I & -\alpha^{2} \gamma^{i 2} I & -\alpha^{2} \gamma^{i 3} I \\
0 & -\delta_{1}{ }^{i} I & -\beta^{i} I & 0 & 0 \\
0 & -\delta_{2}{ }^{i} I & 0 & -\beta^{i} I & 0 \\
0 & -\delta_{3}{ }^{i} I & 0 & 0 & -\beta^{i} I
\end{array}\right) .
$$

Here and in equations (2.28)-(2.29), 0 is the $10 \times 10$ zero matrix and $I$ is the $10 \times 10$ identity matrix. It can be checked easily that the positive definite symmetric $50 \times 50$ matrix

$$
H=\left(\begin{array}{ccccc}
I & 0 & 0 & 0 & 0 \\
0 & \alpha^{-2} I & 0 & 0 & 0 \\
0 & 0 & \gamma^{11} I & \gamma^{12} I & \gamma^{13} I \\
0 & 0 & \gamma^{12} I & \gamma^{22} I & \gamma^{23} I \\
0 & 0 & \gamma^{13} I & \gamma^{23} I & \gamma^{33} I
\end{array}\right)
$$

is a symmetrizer for the system, i.e., $H A^{i}$ are symmetric matrices.

Moreover, system I has only physical characteristic speeds; that is, the eigenvalues of $A^{i} \xi_{i}$ are $\lambda_{+}=-\beta^{i} \xi_{i}+\alpha, \lambda_{0}=-\beta^{i} \xi_{i}$ and $\lambda_{-}=-\beta^{i} \xi_{i}-\alpha$. Let $\eta_{i}$ and $\chi_{i}$ be unit spatial covectors that form an orthonormal triad with $\xi_{i}$, that is, $\eta^{i} \xi_{i}=0=\chi^{i} \xi_{i}=\eta^{i} \chi_{i}$ and $\eta^{i} \eta_{i}=1=\chi^{i} \chi_{i}$, 
where $\eta^{i}=\gamma^{i j} \eta_{j}$ and $\chi^{i}=\gamma^{i j} \chi_{j}$. Let us construct a $50 \times 50$ matrix $V$ whose columns are 50 linearly independent eigenvectors of $A^{i} \xi_{i}$. One such matrix is

$$
V=\left(\begin{array}{ccccc}
0 & I & 0 & 0 & 0 \\
-\alpha I & 0 & 0 & 0 & \alpha I \\
\xi_{1} I & 0 & \eta_{1} I & \chi_{1} I & \xi_{1} I \\
\xi_{2} I & 0 & \eta_{2} I & \chi_{2} I & \xi_{2} I \\
\xi_{3} I & 0 & \eta_{3} I & \chi_{3} I & \xi_{3} I
\end{array}\right)
$$

The first ten columns of $V$ are eigenvectors of $A^{i} \xi_{i}$ with eigenvalue $\lambda_{+}$; the next thirty columns have eigenvalue $\lambda_{0}$; and the last ten columns have eigenvalue $\lambda_{-}$. The characteristic fields in the direction $\xi_{i}$ are obtained from $V^{-1} u$ and are given by $\alpha^{-1} k_{\mu \nu} \pm \xi^{i} d_{i \mu \nu}, g_{\mu \nu}, \eta^{i} d_{i \mu \nu}$ and $\chi^{i} d_{i \mu \nu}$.

\section{System II}

In this section, all indices are lowered and raised by the spatial 3-metric $\gamma_{i j}$ and its inverse $\gamma^{i j}$. The second system presented in this paper is based on a hyperbolic system in [3], which is in turn based on the ADM equations [19]. The system in [3], called system 1, employs the 30 fields $\gamma_{i j}, K_{i j}$, and

$$
d_{k i j}=\partial_{k} \gamma_{i j}
$$

It is obtained by densitizing the lapse and adding multiples of the constraint equations to the evolution equations. The relevant constraints are the Hamiltonian constraint

$$
C=\frac{1}{2}\left({ }^{(3)} R-K_{i j} K^{i j}+K^{2}\right)=0,
$$

the momentum constraints

$$
C_{i}=D_{j} K_{i}^{j}-D_{i} K=0
$$

and the constraint

$$
C_{i j k l}=\partial_{[i} d_{j] k l}=0,
$$

where ${ }^{(3)} R$ and $D_{i}$ are the Ricci scalar and covariant derivative associated with $\gamma_{i j}$, and $K=\gamma^{i j} K_{i j}$.

System 1 has five free parameters that govern how to densitize the lapse and how much of the constraints to add; these parameters determine the system's hyperbolicity. In fact, it has been shown [13] that for a certain range of these parameters, system 1 is symmetrizable hyperbolic and has only physical characteristic speeds.

Here I construct a first-order symmetrizable hyperbolic system based on system 1 that includes the lapse and shift in the system. Let us begin by defining the densitized lapse

$$
Q=\ln \left(\alpha \gamma^{-1 / 2}\right),
$$

where $\gamma=\operatorname{det}\left(\gamma_{i j}\right)$. Next define the new fields

$$
\begin{array}{ll}
Q_{i}=\partial_{i} Q, & Q_{i j}=\partial_{i} \partial_{j} Q, \\
b_{i}{ }^{j}=\partial_{i} \beta^{j}, & b_{i j}{ }^{k}=\partial_{i} \partial_{j} \beta^{k} .
\end{array}
$$

Note that $Q_{i j}=Q_{(i j)}$ and $b_{i j}{ }^{k}=b_{(i j)}{ }^{k}$. The hyperbolic system presented in this section is based on the 70 fields $\gamma_{i j}, K_{i j}, d_{k i j}, Q, Q_{i}, Q_{i j}, \beta^{i}, b_{i}{ }^{j}, b_{i j}{ }^{k}$.

Expressions for time derivatives of these fields are obtained as follows. First, $\partial_{t} \gamma_{i j}$ is obtained from (2.18):

$$
\partial_{t} \gamma_{i j}-\beta^{k} \partial_{k} \gamma_{i j}=-2 \alpha K_{i j}+2 \gamma_{k(i} b_{j)}{ }^{k} .
$$


This is one of the ADM evolution equations with the new fields (3.6) substituted in. Here and henceforth, it is understood that $\alpha$ is to be rewritten in terms of $Q$ using (3.5). Following [3], I add $\zeta_{1} \alpha \gamma_{i j} C$ and $\zeta_{2} \alpha \gamma^{m n} C_{m(i j) n}$ to the second ADM evolution equation (which is equation (2.9) in [3]), where $\zeta_{1}$ and $\zeta_{2}$ are free parameters. Rewriting this equation in terms of the new fields (3.6), we obtain

$$
\begin{aligned}
\partial_{t} K_{i j}=\beta^{k} \partial_{k} & K_{i j}-\frac{1}{2} \alpha \gamma^{m n}\left[\partial_{m} d_{n i j}+2 \partial_{(i} d_{j) m n}-\left(1-\zeta_{2}\right) \partial_{(i} d_{|m n| j)}-\left(1+\zeta_{2}\right) \partial_{m} d_{(i j) n}\right. \\
& \left.-\zeta_{1} \gamma_{i j} \gamma^{k l}\left(\partial_{m} d_{k l n}-\partial_{k} d_{l m n}\right)\right]+2 K_{k(i} b_{j}{ }^{k}-\alpha\left[2 K_{i m} K^{m}{ }_{j}-K K_{i j}+Q_{i j}\right. \\
& +\left(d_{(i j) m}-\frac{1}{2} d_{m i j}\right)\left(\tilde{d}^{m}-d^{m}-Q^{m}\right)+d^{m n}{ }_{i} d_{[n m] j}-\frac{3}{4} d_{i m n} d_{j}{ }^{m n}+Q_{i} Q_{j} \\
& \left.+Q_{(i} d_{j)}+\frac{1}{4} d_{i} d_{j}\right]+\frac{1}{2} \zeta_{1} \alpha \gamma_{i j}\left(\tilde{d}_{m} d^{m}-\tilde{d}_{m} \tilde{d}^{m}-\frac{1}{4} d_{m} d^{m}-\frac{1}{2} d_{k l m} d^{m k l}\right. \\
& \left.+\frac{3}{4} d_{k l m} d^{k l m}-K_{m n} K^{m n}+K^{2}\right),
\end{aligned}
$$

where $d_{i}=\gamma^{j k} d_{i j k}$ and $\tilde{d}_{i}=\gamma^{j k} d_{j k i}$.

Using equality of mixed partials, we have $\partial_{t} d_{k i j}=\partial_{k} \partial_{t} \gamma_{i j}$ which, together with a spatial derivative of (3.7), yields an evolution equation for $d_{k i j}$. Following [3], I add $\zeta_{3} \alpha \gamma_{k(i} C_{j)}$ and $\zeta_{4} \alpha \gamma_{i j} C_{k}$ to this equation and use (3.6) to obtain

$$
\begin{aligned}
\partial_{t} d_{k i j}=\beta^{m} \partial_{m} & d_{k i j}+\alpha \gamma^{m n}\left[\zeta_{3}\left(\gamma_{k(i} \partial_{\mid m} K_{n \mid j)}-\gamma_{k(i} \partial_{j)} K_{m n}\right)+\zeta_{4} \gamma_{i j}\left(\partial_{m} K_{n k}-\partial_{k} K_{m n}\right)\right] \\
& -2 \alpha \partial_{k} K_{i j}+2 \gamma_{m(i} b_{j) k}{ }^{m}+d_{m i j} b_{k}{ }^{m}+2 d_{k m(i} b_{j)}{ }^{m} \\
& -\alpha K_{i j}\left(2 Q_{k}+d_{k}\right)+\alpha \zeta_{4} \gamma_{i j}\left[K_{k m}\left(\frac{1}{2} d^{m}-\tilde{d}^{m}\right)+\frac{1}{2} K^{m n} d_{k m n}\right] \\
& +\alpha \zeta_{3}\left[\gamma_{k(i} K_{j) m}\left(\frac{1}{2} d^{m}-\tilde{d}^{m}\right)+\frac{1}{2} K^{m n} \gamma_{k(i} d_{j) m n}\right],
\end{aligned}
$$

where $\zeta_{3}$ and $\zeta_{4}$ are free parameters. The parameters $\left(\zeta_{1}, \zeta_{2}, \zeta_{3}, \zeta_{4}\right)$ in the above equations correspond to the parameters $(\gamma, \zeta, \eta, \chi)$ in [3]. The parameter $\sigma$ in [3] has been set to $1 / 2$ by the definition (3.5).

The next step is to specify evolution equations for the lapse density and shift. Spatial derivatives of these equations will then yield evolution equations for the fields (3.6). I consider a particular form for the lapse density and shift evolution equations, a form that results in a symmetrizable hyperbolic system but yet allows four functions of spacetime to be freely specified. The equations are

$$
\begin{aligned}
& \partial_{t} Q-\beta^{i} \partial_{i} Q=\psi^{0}\left(t, x^{j} ; Q\right), \\
& \partial_{t} \beta^{i}-\beta^{j} \partial_{j} \beta^{i}=\psi^{i}\left(t, x^{k} ; Q, \beta^{m}\right),
\end{aligned}
$$

where $\psi^{\mu}$ are arbitrary but predetermined functions of space, time and lapse density (and of shift in the case of $\psi^{i}$ ).

Evolution equations for the fields (3.6) are obtained by taking spatial derivatives of (3.10) and (3.11), and using equality of mixed partials. For example, $\partial_{t} Q_{i}=\partial_{i} \partial_{t} Q=$ $\partial_{i}\left(\beta^{j} \partial_{j} Q+\psi^{0}\right)$. We obtain

$$
\begin{aligned}
& \partial_{t} Q_{i}-\beta^{j} \partial_{j} Q_{i}=Q_{j} b_{i}{ }^{j}+\partial_{i} \psi^{0}, \\
& \partial_{t} Q_{i j}-\beta^{k} \partial_{k} Q_{i j}=2 Q_{k(i} b_{j)}{ }^{k}+Q_{k} b_{i j}{ }^{k}+\partial_{i} \partial_{j} \psi^{0}, \\
& \partial_{t} \beta_{i}{ }^{j}-\beta^{k} \partial_{k} \beta_{i}{ }^{j}=b_{i}{ }^{k} b_{k}{ }^{j}+\partial_{i} \psi^{j}, \\
& \partial_{t} b_{i j}{ }^{k}-\beta^{m} \partial_{m} b_{i j}{ }^{k}=2 b_{(i}{ }^{m} b_{j) m}{ }^{k}+b_{i j}{ }^{m} b_{m}{ }^{k}+\partial_{i} \partial_{j} \psi^{k},
\end{aligned}
$$

where it is understood that the spatial derivatives of $\psi^{\mu}$ are to be written, using (3.6), in terms of fields only and not derivatives of fields. 
When the system (3.7)-(3.15), called system II, is put in the form (2.2) with $u=$ $\left(\gamma_{i j}, K_{i j}, d_{k i j}, Q, Q_{i}, Q_{i j}, \beta^{i}, b_{i}{ }^{j}, b_{i j}{ }^{k}\right)^{T}$, the $70 \times 70$ matrices $A^{i}$ have the block diagonal form

$$
A^{i}=\left(\begin{array}{cc}
\tilde{A}_{30 \times 30}^{i} & 0_{30 \times 40} \\
0_{40 \times 30} & -\beta^{i} I_{40 \times 40}
\end{array}\right) .
$$

The nontrivial parts $\tilde{A}^{i}$ of $A^{i}$ come from the evolution equations (3.7)-(3.9) for the 30 fields $\gamma_{i j}, K_{i j}, d_{k i j}$. Since the principal parts of these equations are identical (after relabelling the free parameters as indicated above) to the principal parts of the system 1, evolution equations for $\gamma_{i j}, K_{i j}, d_{k i j}$ given in [3], the matrices $\tilde{A}^{i}$ are identical to the corresponding matrices in [3]. This implies that if system 1 is symmetrizable, so is system II. Indeed, the matrix

$$
H=\left(\begin{array}{cc}
\tilde{H}_{30 \times 30} & 0_{30 \times 40} \\
0_{40 \times 30} & I_{40 \times 40}
\end{array}\right)
$$

where $\tilde{H}_{30 \times 30}$ symmetrizes system 1 , is a symmetrizer for system II. In other words, if the $30 \times 30$ matrices $\tilde{H} \tilde{A}^{i}$ are symmetric, then so are the $70 \times 70$ matrices $H A^{i}$. In addition, the characteristic fields $Q, Q_{i}, Q_{i j}, \beta^{i}, b_{i}{ }^{j}, b_{i j}{ }^{k}$ all propagate normal to the time slices.

It has been shown [13] that system 1 in [3] is symmetrizable and has only physical characteristic speeds when the free parameters are chosen as follows:

$$
\begin{array}{ll}
\zeta_{3}=\frac{-8}{5+10 \zeta_{1}+7 \zeta_{2}+6 \zeta_{1} \zeta_{2}}, & -5 / 3<\zeta_{2}<0, \\
\zeta_{4}=-\frac{4+10 \zeta_{1}+4 \zeta_{2}+6 \zeta_{1} \zeta_{2}}{5+10 \zeta_{1}+7 \zeta_{2}+6 \zeta_{1} \zeta_{2}}, & 5+10 \zeta_{1}+7 \zeta_{2}+6 \zeta_{1} \zeta_{2} \neq 0 .
\end{array}
$$

We conclude that for the same choice of parameters, system II is symmetrizable and has only physical characteristic speeds.

System II is not practical for numerical implementation. Since the lapse density and shift evolution equations (3.10) and (3.11) decouple from the rest of the system, they can be evolved separately to obtain the lapse density and shift as spacetime functions. These functions can then be substituted into system 1 in [3]. Therefore, the full seventy-field system II does not need to be evolved; the thirty-field system 1 suffices.

However, system II is useful from a theoretical point of view. Consider a solution of Einstein's equations in an arbitrary gauge. Using the densitized lapse and shift from this solution, compute the left-hand sides of equations (3.10) and (3.11). Set the spacetime functions $\psi^{\mu}$ equal to these computed quantities. Take initial values for the fields in system II from the spacetime metric under consideration. System II can now be used, with these initial values and with $\psi^{\mu}$ as defined above, to obtain the entire metric by evolving hyperbolic equations that are part of a symmetrizable system with only physical characteristic speeds. So system II can be used to obtain any solution of Einstein's equations in any gauge using hyperbolic evolution for the entire metric, including the densitized lapse and shift. Note, however, that the lapse is not evolved directly in this system; it is obtained from the densitized lapse via equation (3.5).

\section{Future directions}

An important future research direction is to study and understand the stability of numerical implementations of system I. It has been shown in previous work [3] that some hyperbolic systems are more stable than others when used to simulate black holes in three spatial dimensions. The reasons for this behaviour are not yet understood. Another future research 
direction is to explore how to use the free functions $f^{\mu}$ in system I to control the coordinate system.

\section{Acknowledgments}

I am grateful to Lee Lindblom and Mark Scheel for valuable discussions and for sharing their results before publication, and to Olivier Sarbach, Kip Thorne and Manuel Tiglio for useful comments on the manuscript. This research was supported in part by NSF grant PHY-9900776.

\section{References}

[1] Friedrich H and Rendall A D 2000 Lect. Notes Phys. 540127

[2] Reula O A 1998 Liv. Rev. Rel. 1998-3

[3] Kidder L E, Scheel M A and Teukolsky S A 2001 Phys. Rev. D 64064017

[4] Fischer A E and Marsden J E 1972 Commun. Math. Phys. 281

[5] Friedrich H 1985 Commun. Math. Phys. 100525

[6] Friedrich H 1996 Class. Quantum Grav. 131451

[7] Anderson A and York J W 1999 Phys. Rev. Lett. 824384

[8] Smarr L and York J W 1978 Phys. Rev. D 172529

[9] Brady P R, Creighton J D E and Thorne K S 1998 Phys. Rev. D 58061501

[10] Garfinkle D, Gundlach C, Isenberg J and ÓMurchadha N 2000 Class. Quantum Grav. 173899

[11] Bona C, Massó J, Seidel E and Stela J 1995 Phys. Rev. Lett. 75600

[12] Alcubierre M, Brügmann B, Pollney D, Seidel E and Takahashi R 2001 Phys. Rev. D 64 061401(R)

[13] Lindblom L and Scheel M A 2002 Preprint gr-qc/0206035

[14] Misner C W, Thorne K S and Wheeler J A 1973 Gravitation (San Francisco, CA: Freeman)

[15] York J W 1978 Sources of Gravitational Radiation ed L Smarr (New York: Cambridge University Press) p 83

[16] Bruhat Y 1962 Gravitation: an Introduction to Current Research ed L Witten (New York: Wiley) p 130

[17] Garfinkle D 2002 Phys. Rev. D 65044029

[18] Fock V 1964 The Theory of Space, Time and Gravitation (New York: MacMillan)

[19] Arnowitt R, Deser S and Misner C W 1962 Gravitation: an Introduction to Current Research ed L Witten (New York: Wiley) p 227 